Punto de vista

\title{
Modulación hormonal del donante de órganos. Utilidad de los esteroides
}

\author{
JUAN C. MICHELENAa , CARLOS CHAMORRO ${ }^{b}$, JUAN A. FALCÓN Y SANDRA GARCÉSa \\ aCoordinación Nacional de Trasplantes de la República de Cuba. Cuba. \\ bServicio de Medicina Intensiva. Hospital Puerta de Hierro. Coordinación Autonómica de Trasplantes de la Comunidad \\ de Madrid. Madrid. España. \\ 'Dirección Nacional del Sistema integrado de Urgencias Médicas de la República de Cuba. Cuba.
}

Recientemente el grupo de trabajo compuesto por la Organización Nacional de Trasplantes (ONT), la Sociedad Española de Medicina Intensiva, Crítica y de Unidades Coronarias (SEMICYUC) y otras sociedades científicas han recomendado la administración de $15 \mathrm{mg} / \mathrm{kg}$ de metilprednisolona en la estrategia de manejo del donante pulmonar. Esta recomendación está basada en estudios descriptivos y retrospectivos. La revisión de las publicaciones en el ámbito experimental y clínico también indica un potencial beneficio de los esteroides en el manejo del donante tanto de órganos intratorácicos como abdominales. La administración de esteroides apenas instaurada la muerte encefálica puede inhibir la liberación o prevenir las alteraciones que producen las citocinas proinflamatorias, puede estabilizar las membranas celulares, reducir la expresión de las moléculas de adhesión e interferir en la peroxidación lipídica que ocurre después de la isquemia. Sería recomendable realizar estudios prospectivos y comparativos para demostrar la utilidad de este tratamiento. Hasta la realización de estos estudios, y conociendo la repercusión nociva de la actividad inflamatoria en relación con la muerte encefálica en los órganos a trasplantar, recomendamos utilizar, tan pronto como sea posible, $15 \mathrm{mg} / \mathrm{kg}$ de metilprednisolona en el donan-

Este trabajo forma parte de las tesinas realizadas por los Dres. Michelena y Falcón, y dirigidas por el Dr. Chamorro, durante el máster Alianza en Donación y Trasplante de Órganos, Tejidos y Células, organizado por la ONT.

Correspondencia: Dr. C. Chamorro.

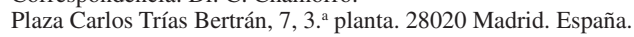

Correo electrónico: cchamorro.hpth@salud.madrid.org

Manuscrito aceptado el 9-12-2008. te de cualquier órgano. Sus potenciales efectos beneficiosos inmunomoduladores, así como su bajo coste y la ausencia de efectos adversos, justifican esta recomendación.

PALABRAS CLAVE: Muerte encefálica. Disfunción orgánica. Donación. Esteroides. Mantenimiento. Inmunomodulación.

\section{HORMONE MODULATION OF ORGAN DONOR. UTILITY OF THE STEROIDS}

Recently, the work group made up of the National Transplant Organization (Organización Nacional de Trasplantes, ONT), Spanish Society of Intensive, Critical Medicine and Coronary Units (Sociedad Española de Medicina Intensiva, Crítica y de Unidades Coronarias, SEMICYUC) and other Scientific Societies have recommended using 15 $\mathrm{mg} / \mathrm{kg}$ of methyl prednisolone during the management of lung donors after brain death. This recommendation is based on descriptive and retrospective studies. However, the review of different experimental and clinical studies also suggests a potential benefit of using steroids in either thoracic or abdominal organ donors during management strategies. In brain death management, early steroid administration may decrease cytokine production and also may prevent alterations induced by proinflammatoy mediators, stabilize cell membranes, reduce expression of cell surface adhesion molecules and avoid lipid peroxidation after the ischemic period. This could be beneficial in increasing number and quality of organs harvested and in decreasing rejection episodes after transplant. It would be very recommendable to carry out prospective and comparative studies to demonstrate these potential utilities. Meanwhile and knowing the deleterious effects of 
inflammatory activity arising during and after brain death, we recommend using $15 \mathrm{mg} / \mathrm{kg}$ of methyl prednisolone in the organ donor management, as soon as possible. The potential benefit of its immunomodulation effects, its low cost and the absence of major side effects can justify this recommendation.

KEY WORDS: Brain death. Organ donor management. Immunomodulation. Cytoquine production. Steroids. Methyl-prednisolone.

\section{INTRODUCCIÓN}

Durante la muerte encefálica (ME) se producen una serie de trastornos hemodinámicos, hormonales e inflamatorios que pueden alterar o dañar irreversiblemente la función de los diferentes órganos. En unos casos, estos cambios pueden impedir el aprovechamiento del órgano severamente dañado y, en otros, las alteraciones producidas pueden favorecer la aparición de fenómenos de rechazo en el receptor ${ }^{1}$. El proceso de mantenimiento del donante de órganos es fundamental para impedir, aminorar o incluso revertir estas alteraciones ${ }^{2}$. Diferentes sociedades científicas han publicado recomendaciones de manejo del donante de órganos ${ }^{3-5}$. Muchas de ellas están dirigidas, específicamente, al mantenimiento del donante de órganos intratorácicos, ya que el corazón y el pulmón son los órganos más afectados durante la instauración de la muerte encefálica. Sólo entre un 10 y un $20 \%$ de los donantes llegan a donar el pulmón o el corazón. Las estrategias de aumento en la donación de órganos intratorácicos consisten en liberalizar los criterios de selección e intentar recuperar órganos que en la primera valoración son considerados no útiles. Dentro de las recomendaciones promulgadas para recuperar órganos «no válidos» destaca el concepto de la modulación hormonal. La administración de hormonas tiroideas, vasopresina y esteroides podría aumentar la cantidad y la calidad de los órganos intratorácicos extraídos $^{6-8}$. Sin embargo, recientemente, diferentes revisiones de la literatura muestran la escasa evidencia científica en la que se soporta la administración de hormonas tiroideas ${ }^{9,10}$. Diferentes sociedades científicas internacionales, así como el Grupo de Trabajo formado por la Organización Nacional de Trasplantes (ONT) en colaboración con la Sociedad Española de Medicina Intensiva, Crítica y de Unidades Coronarias (SEMICYUC) y otras sociedades científicas nacionales, recomiendan la administración de $15 \mathrm{mg} / \mathrm{kg}$ de metilprednisolona en la estrategia de manejo del donante pulmonar ${ }^{11}$. El objetivo de este trabajo es revisar las publicaciones sobre el uso y la utilidad de los esteroides en el manejo del donante de órganos.

\section{MATERIAL Y MÉTODO}

Revisión bibliográfica de los estudios publicados sobre el empleo de esteroides en el manejo de donante de órganos. Los trabajos fueron obtenidos de la base electrónica de datos MEDLINE a través de PubMed con las búsquedas aisladas o combinadas: «steroids and brain death», «steroids and organ donor management», «steroids and organ donor treatment», «steroid and organ procurement», «steroids and lung donor», «brain death and organ dysfunction», «organ donor and hormonal therapy» $\mathrm{y}$ «organ donor management». En los trabajos seleccionados se revisó la bibliografía para identificar estudios clínicos o experimentales que pudieran aportar o clarificar aspectos importantes.

\section{RESULTADOS}

Hemos identificado 7 estudios que valoran directa o indirectamente la repercusión, en los diferentes órganos, del uso de esteroides en el manejo del donante, 2 de ellos son clínicos ${ }^{12,13}, 3$ son estudios retrospectivos sobre series históricas ${ }^{14-16}$ y 2 son experimenta$\operatorname{les}^{17,18}$. Los 2 estudios clínicos publicados valoran específicamente el uso de esteroides en el manejo del donante de órganos, ambos en el seno del tratamiento del donante pulmonar. Follette et al ${ }^{12}$, en 1998, publicaron un estudio retrospectivo sobre la repercusión del uso de altas dosis de metilprednisolona $(15 \mathrm{mg} /$ $\mathrm{kg}$ ) en el potencial donante pulmonar. Los autores revisaron y compararon de forma retrospectiva la repercusión de la administración de esteroides en la oxigenación, valorada por el cociente $\mathrm{PaO}_{2} / \mathrm{FiO}_{2}$, en 80 potenciales donantes de pulmón. El grupo control fue una serie contemporánea de 38 potenciales donantes de pulmón en los que no se administró este fármaco. La decisión de tratar o no con esteroides fue discrecional por el coordinador hospitalario de trasplantes y se realizó a las $16 \pm 1 \mathrm{~h}$ tras el enclavamiento cerebral, los donantes estuvieron una media de $16 \pm 1 \mathrm{~h}$ bajo la influencia de este tratamiento. Los potenciales donantes que no recibieron el fármaco presentaron, durante su mantenimiento, un descenso progresivo del cociente $\mathrm{PaO}_{2} / \mathrm{FiO}_{2}$ (cambio de -34) y en los tratados, se observó un ascenso $(+16)$, estos cambios mostraron significación estadística $(\mathrm{p}=0,012)$. Sólo el $8 \%$ de los no tratados llegaron a donar los pulmones frente al $31 \%$ de los tratados $(p=0,005)$. Los autores concluyeron acerca de la utilidad del uso de esteroides en este tipo de donantes.

El segundo estudio publicado fue el de Venkateswaran et $\mathrm{al}^{13} \mathrm{en} 2008$. El estudio es prospectivo, aleatorizado, comparativo y ciego sobre la utilidad de la administración de triyodotironina (T3) o esteroides en los potenciales donantes de órganos intratorácicos. Los autores aleatorizan de forma ciega a 4 grupos de tratamiento; sólo T3 (17 donantes), sólo esteroides, $1 \mathrm{~g}$ de metilprednisolona (MP) (15 donantes), T3 y MP (14 donantes) y placebo (14 donantes). Además, personal específico manejó a todos estos donantes de acuerdo con un estricto protocolo de monitorización y tratamiento, consistente en monitorización hemodinámica con catéteres de Swan-Ganz y PiCCO, manejo adecuado del volumen intratorácico y del agua extrapulmonar (EVLW), control y normalización de la presión venosa central (PVC), el gasto 
cardíaco (CO) y las resistencias vasculares sistémicas (RVS). Asimismo, se realizaron frecuentes maniobras de aspiración de secreciones con broncoscopia precoz y a veces repetida, así como maniobras de reclutamiento. Además, los autores compararon los resultados de este protocolo con una serie contemporánea de potenciales donantes de órganos intratorácicos a quienes, por unas razones u otras, no se pudo incluir en el protocolo de estudio. El inicio del tratamiento activo se produjo a las $12,5 \pm 8,1 \mathrm{~h}$ del enclavamiento $\mathrm{y}$ fueron tratados una media de $7 \pm 1,2 \mathrm{~h}$. Los autores no encontraron ninguna diferencia en cuanto a la función pulmonar en los tratados con T3, MP o placebo. En un análisis posterior, los autores encontraron que los potenciales donantes tratados con MP, previamente a la extracción, tenían EULW (o agua pulmonar extrapulmonar indexada [EUKWI]) más baja que los no tratados. Hubo más donación de pulmones entre los potenciales donantes incluidos en el protocolo activo de tratamiento que en los no incluidos (el 43 frente al 29\%). Los autores concluyen que la administración de T3 y MP no afecta negativamente al mantenimiento del donante intratorácico y que la MP podría tener efectos beneficiosos al evitar la acumulación de agua.

Hay otro estudio publicado que apoya la tesis de administrar MP a los donantes potenciales de pulmón. McElhinney et $\mathrm{al}^{14}$ realizaron, en 2001, un estudio retrospectivo para analizar los factores que pudieran haber influido en el potencial donante intratorácico para llegar a ser donante efectivo de pulmón. De los diferentes factores analizados (demográficos, clínicos, exploratorios y de manejo) en 251 potenciales donantes, sólo 2 fueron significativos en el análisis multivariable: que tenían auscultación pulmonar normal (odds ratio $[\mathrm{OR}]=2,1[1,3-3,4] ; \mathrm{p}=$ $0,008)$ y que habían recibido metilprednisolona en el mantenimiento (OR $=3$ [1,9-4,9]; $\mathrm{p}<0,001)$.

La influencia del uso de esteroides en la contractilidad cardíaca del donante está contemplada en 2 estudios experimentales realizados por un mismo grupo. En 2005 , Lyons et al ${ }^{17}$ realizaron un estudio aleatorizado en cerdos sometidos a situación de muerte encefálica. Realizaron 3 grupos de tratamiento; un grupo control (5 casos), un grupo tratado con $15 \mathrm{mg} /$ $\mathrm{kg}$ de MP administrado $2 \mathrm{~h}$ antes de la instauración de la ME ( 5 casos) y otro grupo tratado con MP 1 h después de la ME (5 casos). Los autores demostraron que el uso de MP preservaba la función ventricular después de la ME, tanto de los parámetros de contracción como de relajación ventricular. La administración previa a la muerte encefálica conllevó mejores parámetros hemodinámicos que la administración después de la ME. Estos mismos autores, 2 años después, demostraron, en el mismo modelo experimental, que el uso de MP conlleva un descenso de la liberación de mediadores proinflamatorios, en especial del factor de necrosis tumoral alfa $(\mathrm{TNF} \alpha)$ e interleucina 6 (IL-6), así como de las moléculas de adhesión celular (ICAM-1). Este descenso fue muy superior si el tratamiento esteroideo se iniciaba antes del desarrollo de la $\mathrm{ME}^{18}$.
La influencia de los esteroides en el funcionamiento renal tras la muerte encefálica sólo está contemplada en un estudio experimental. Pratschke et al ${ }^{19}$ realizaron, en 2001, un estudio sobre ratas sometidas a muerte encefálica. Administraron $10 \mathrm{mg} / \mathrm{kg}$ de MP en el mismo momento de la instauración de la ME a 10 de ellas y a otras 10, una sustancia que inhibe la adhesión celular de los leucocitos (soluble P-selectin glycoprotein ligand [sPSGL]), otras 12 no tratadas fueron el grupo control. Los autores demostraron, tras el estudio anatomopatológico, que la administración de los fármacos de estudio disminuyó la intensidad de la inflamación en relación con los fenómenos de isquemia-reperfusión, la incidencia de rechazo precoz y la supervivencia del injerto.

\section{DISCUSIÓN}

Los esteroides, en particular la administración de $15 \mathrm{mg} / \mathrm{kg}$ de metilprednisolona, son recomendados por diferentes autores ${ }^{6,20-23}$ y sociedades científicas ${ }^{3,5}$ para el manejo del donante de órganos, en especial del donante de pulmón y de corazón inicialmente disfuncionantes. Recientemente el Grupo de Trabajo conjunto de la ONT, la SEMICYUC y otras sociedades científicas ha recomendado la administración de este fármaco para el mantenimiento del donante de pulmón ${ }^{11}$.

Sin embargo, como se demuestra en esta revisión, hay muy pocos estudios que demuestren irrefutablemente esta recomendación. El trabajo de Follete et $\mathrm{al}^{12}$, publicado en 1998 , es el primero en que se indica que la administración de esteroides puede ser beneficiosa y es el estudio en que se apoyan los diferentes autores para recomendar su utilización. Sin embargo, este estudio tiene muchos déficit metodológicos. Es de diseño retrospectivo y, probablemente, su mayor handicap es que la decisión de administrar esteroides fue discrecional por parte del coordinador de trasplantes. Tal vez esto implica un sesgo, ya que el estudio puede identificar una serie de donantes en cuyo manejo estuvo un coordinador con mayor preocupación en el cuidado y el tratamiento activo del potencial donante. Diferentes autores han demostrado que la incorporación de un protocolo estricto de mantenimiento, en el que se incluyen medidas de ventilación mecánica, realización de broncoscopias para limpieza del árbol bronquial, maniobras de reclutamiento alveolar y estricto control del balance hídrico, puede aumentar la donación pulmonar hasta en un 30\% $\%^{13,24-26}$. Algunos de estos protocolos han incluido la administración de metilprednisolona ${ }^{13,25}$, pero otros, en cambio, no administraron esteroides y tuvieron los mismos resultados 24,26 .

Hasta el año 2008 no se ha publicado otro estudio que intente demostrar la utilidad de los esteroides. Sin embargo, Venkateswaran et $\mathrm{al}^{13}$, en un estudio de mayor rigor científico, prospectivo, aleatorizado, comparativo y ciego, no han confirmado los hallazgos de Follete et al en la mejoría de la oxigenación. Sólo en un análisis post-hoc los autores han demostrado que los potenciales donantes de pulmón trata- 
dos con MP tenían menos agua intrapulmonar que los no tratados y esto pudiera haber influido en la mayor tasa de donación pulmonar.

La utilidad de los esteroides en el donante de órganos se sostiene desde un punto de vista teórico. Durante la muerte encefálica, además de cambios hemodinámicos y hormonales, se produce una elevación de citocinas proinflamatorias, como TNF $\alpha$, IL-1 e IL-6. Este medio inflamatorio puede dañar los órganos o inducir cambios inmunológicos celulares que producen la conocida mayor incidencia de fenómenos de rechazo en los receptores de órganos procedente de donantes en ME que de donantes vivos ${ }^{27,28}$. El pulmón y el corazón son, probablemente, los órganos más dañados desde el punto de vista funcional durante el desarrollo de la ME. Factores como la contusión pulmonar, el edema neurogénico, la broncoaspiración y el daño pulmonar o la neumonía en relación con la ventilación justifican que sólo uno de cada 5-6 sujetos sea donante de pulmones. Además, como demostraron Fisher et $\mathrm{al}^{29}$, los cambios inflamatorios producidos en la ME afectan de forma muy negativa al pulmón. Estos autores demostraron un aumento de la infiltración por neutrófilos y un incremento significativo de los valores de IL-8 en el lavado broncoalveolar de los pulmones de fallecidos en situación de ME. Este hecho se puede asociar a una mayor incidencia de disfunción precoz del injerto y de mortalidad precoz en el receptor ${ }^{30,31}$. Estos cambios también afectan negativamente al corazón, el hígado, los riñones y el intestino. El corazón y los demás órganos de los fallecidos en ME frecuentemente tienen cambios inflamatorios secundarios a una combinación de los fenómenos de isquemia-reperfusión y del medio inflamatorio que se produce en el seno de la muerte encefálica. La activación del sistema inmunitario aumenta la inmunogenicidad de los órganos, y puede causar un aumento en la incidencia de rechazo precoz, así como afectar negativamente a la supervivencia a medio y largo plazo del órgano trasplantado ${ }^{32-37}$.

La administración precoz de esteroides en la instauración de la muerte encefálica puede inhibir la liberación o prevenir las alteraciones que producen las citocinas proinflamatorias, puede estabilizar las membranas celulares, reducir la expresión de las moléculas de adhesión e interferir en la peroxidación lipídica que ocurre después de la isquemia ${ }^{38,39}$. Kuecueck et $\mathrm{al}^{40}$ demostraron que la administración de esteroides en el donante conllevó un descenso significativo de las citocinas inflamatorias, tanto local como en sangre, en los diferentes órganos extraídos. Estos efectos pueden aumentar el número de órganos válidos para su extracción, así como podrían disminuir la incidencia de fracaso precoz del injerto y de episodios posteriores de rechazo. Un reciente estudio demuestra que la administración de esteroides y no de hormonas tiroideas conlleva un aumento en el número de órganos válidos ${ }^{15}$. Un análisis retrospectivo, realizado por la United Network for Organ Sharing (UNOS) sobre 14.616 trasplantados de riñón de cadáver, demostró que la administración de esteroides en el donante causaba un descenso del $10 \%$ en la incidencia de la pérdida del injerto renal al año de su implante ${ }^{16}$. Además, en el caso de los pulmones, la administración de esteroides podría aumentar el aclaramiento del fluido alveolar, tal y como se indica en diferentes estudios experimentales ${ }^{41,42}$.

En resumen, la recomendación de uso de esteroides en el protocolo de manejo del donante de órganos, en particular del donante de pulmón, está basada en estudios descriptivos y retrospectivos. Sería recomendable la realización de estudios prospectivos y comparativos para demostrar la utilidad. Hasta la realización de estos estudios, y conociendo la repercusión nociva de la actividad inflamatoria en relación con la muerte encefálica en los órganos a trasplantar, recomendamos la utilización, tan pronto como sea posible, de $15 \mathrm{mg} / \mathrm{kg}$ de metilprednisolona en el donante de cualquier órgano. Sus potenciales efectos beneficiosos inmunomoduladores, así como su bajo coste y la ausencia de efectos adversos, pueden justificar esta recomendación.

\section{BIBLIOGRAFÍA}

1. Pratschke J, Wilhelm MJ, Kusaka M, Basker M, Cooper DK Hancock WW, et al. Brain death and its influence on donor organ quality and outcome after transplantation. Transplantation. 1999;67:343-8.

2. Salim A, Martin M, Brown C, Belzberg H, Rhee P, Demetriades D. Complications of brain death: frequency and impact on organ retrieval. Am Surg. 2006;72:377-81.

3. Shemie SD, Ross H, Pagliarello J, Baker AJ, Greig PD, Brand $\mathrm{T}$, et al. Organ donor management in Canada: recommendations of the forum on Medical Management to Optimize Donor Organ Potential. CMAJ. 2006;174:S13-32.

4. Boulard G, Guiot P, Pottecher T, Tenaillon A. Management of brain-dead subjets for organ harvesting. Organ and Tissues. 2005;3:185-92.

5. Zaroff JG, Rosengard BR, Armstrong WF, Babcock WD, D'Alessandro A, Dec GW, et al. Consensus conference report: maximizing use of organs recovered from the cadaver donor: cardiac recommendations, March 28-29, 2001, Crystal City, Va. Circulation. 2002;106:836-41.

6. Novitzky D, Cooper DK, Rosendale JD, Kauffman HM Hormonal therapy of the brain-dead organ donor: experimental and clinical studies. Transplantation. 2006;82:1396-401.

7. Salim A, Velmahos GC, Brown C, Belzberg H, Demetriades D. Aggressive organ donor management significantly increases the number of organs available for transplantation. J Trauma. 2005;58:991-4.

8. Rosendale JD, Kauffman HM, McBride MA, Chabalewski FL, Zaroff JG, Garrity ER, et al. Aggressive pharmacologic donor management results in more transplanted organs. Transplantation. 2003;75:482-7.

9. Falcon JA, Chamorro C, Peraza V, Michelena JC. Mitos o realidades con el uso de hormonas tiroideas en el manejo del donante de órganos. Rev Esp Trasp. 2007;16:216-26.

10. Powner DJ, Hernandez M. A review of thyroid hormone administration during adult donor care. Prog Transplant. 2005;15:2027.

11. Organización Nacional de Trasplantes. Protocolo de manejo del donante torácico: Estrategias para mejorar el aprovechamiento de órganos. Documento de consenso, 2006. Disponible en: http:// www.semicyuc.org/?q=node/212

12. Follette DM, Rudich SM, Babcock WD. Improved oxygenation and increased lung donor recovery with high-dose steroid administration after brain death. J Heart Lung Transplant. 1998; 17:423-9.

13. Venkateswaran RV, Patchell VB, Wilson IC, Mascaro JG, Thompson RD, Quinn DW, et al. Early donor management increa- 
ses the retrieval rate of lungs for transplantation. Ann Thorac Surg. 2008;85:278-86

14. McElhinney DB, Khan JH, Babcock WD, Hall TS. Thoracic organ donor characteristics associated with successful lung procurement. Clin Transplant. 2001;15:68-71.

15. Selck FW, Deb P, Grossman EB. Deceased organ donor characteristics and clinical interventions associated with organ yield Am J Transplant. 2008;8:965-74.

16. McBride MA, Peters TG, Henderson JM, et al. Expanded donor study. UNOS Report to the Health Resources \& Services Administration; 1997. p. 17-34.

17. Lyons JM, Pearl JM, McLean KM, Akhter SA, Wagner CJ, Pandalai PK, et al. Glucocorticoid administration reduces cardiac dysfunction after brain death in pigs. J Heart Lung Transplant. 2005;24:2249-54.

18. McLean KM, Duffy JY, Pandalai PK, Lyons JM, Bulcao CF, Wagner CJ, et al. Glucocorticoids alter the balance between proand anti-inflammatory mediators in the myocardium in a porcine model of brain death. J Heart Lung Transplant. 2007;26: 78-84.

19. Pratschke J, Kofla G, Wilhelm MJ, Vergopoulos A, Laskowski I, Shaw GD, et al. Improvements in early behavior of rat kidney allografts after treatment of the brain-dead donor. Ann Surg. 2001;234:732-40.

20. Kutsogiannis DJ, Pagliarello G, Doig C, Ross H, Shemie SD. Medical management to optimize donor organ potential: review of the literature. Can J Anaesth. 2006;53:820-30.

21. Wood KE, Becker BN, McCartney JG, D'Alessandro AM, Coursin DB. Care of the potential organ donor. N Engl J Med. 2005;351:2730-9.

22. De Perrot M, Snell GI, Babcock WD, Meyers BF, Patterson G, Hodges TN, et al. Strategies to optimize the use of currently available lung donors. J Heart Lung Transplant. 2004;23:1127-34

23. Avlonitis VS, Fisher AJ, Kirby JA, Dark JH. Pulmonary transplantation: the role of brain death in donor lung injury. Transplantation. 2003;75:1928-33.

24. Gabbay E, Williams TJ, Griffiths AP, Macfarlane LM, Kotsimbos TC, Esmore DS, et al. Maximizing the utilization of donor organs offered for lung transplantation. Am J Respir Crit Care Med. 1999;160:265-71.

25. Straznicka M, Follette DM, Eisner MD, Roberts PF, Menza RL, Babcock WD. Aggressive management of lung donors classified as unacceptable: excellent recipient survival one year after transplantation. J Thorac Cardiovasc Surg. 2002;124:250-8.

26. Angel LF, Levine DJ, Restrepo MI, Johnson S, Sako E, Carpenter A, et al. Impact of a lung transplantation donor-management protocol on lung donation and recipient outcomes. Am J Respir Crit Care Med. 2006;174:710-6.

27. Takada M, Nadeau KC, Hancock WW, Mackenzie HS, Shaw GD, Waaga AM, et al. Effects of explosive brain death on cytokine activation of peripheral organs in the rat. Transplantation. 1998;65:1533-42.

28. Lopau K, Mark J, Schramm L, Heidbreder E, Wanner C. Hormonal changes in brain death and immune activation in the donor. Transpl Int. 2000;13 Suppl 1:S282-5.
29. Fisher AJ, Donnelly SC, Hirani N, Burdick MD, Strieter RM, Dark JH, et al. Enhanced pulmonary inflammation in organ donors following fatal non-traumatic brain injury. Lancet. 1999;353:1412-3.

30. Avlonitis VS, Wigfield CH, Kirby JA, Dark JH. The hemodynamic mechanisms of lung injury and systemic inflammatory response following brain death in the transplant donor. Am J Transplant. 2005;5:684-93.

31. Fisher AJ, Donnelly SC, Hirani N, Haslett C, Strieter RM, Dark JH, et al. Elevated levels of interleukin-8 in donor lungs is associated with early graft failure after lung transplantation. Am J Respir Crit Care Med. 2001;163:259-65.

32. Wilhelm MJ, Pratschke J, Beato F, Taal M, Laskowski IA, Paz DM, et al. Activation of proinflammatory mediators in heart transplants from brain-dead donors: evidence from a model of chronic rat cardiac allograft rejection. Transplant Proc. 2002;34:2359-60.

33. Wilhelm MJ, Pratschke J, Beato F, Taal M, Kusaka M, Hancock WW, et al. Activation of the heart by donor brain death accelerates acute rejection after transplantation. Circulation. 2000;102:2426-33.

34. Weiss S, Kotsch K, Francuski M, Reutzel-Selke A, Mantouvalou L, Klemz R, et al. Brain death activates donor organs and is associated with a worse $\mathrm{I} / \mathrm{R}$ injury after liver transplantation. Am J Transplant. 2007;7:1584-93.

35. Olinga P, Van der Hoeven JA, Merema MT, Freund RL, Ploeg RJ, Groothuis GM. The influence of brain death on liver function. Liver Int. 2005;25:109-16.

36. Pratschke J, Wilhelm MJ, Laskowski I, Kusaka M, Beato F, Tullius SG, et al. Influence of donor brain death on chronic rejection of renal transplants in rats. J Am Soc Nephrol. 2001;12:247481

37. Koudstaal LG, 't Hart NA, Van den Berg A, Olinga P, Van Goor H, Ploeg RJ, et al. Brain death causes structural and inflammatory changes in donor intestine. Transplant Proc. 2005;37:4489.

38. Dupont E, Schandene L, Denys C, Wybran J. Differential in vitro actions of cyclosporin, methylprednisolone, and 6-mercaptopurine: implications for drugs' influence on lymphocyte activation mechanisms. Clin Immunol Immunopathol. 1986;40:422-8.

39. Wan S, DeSmet JM, Antoine M, Goldman M, Vincent JL, LeClerc JL. Steroid administration in heart and heart-lung transplantation: is the timing adequate? Ann Thorac Surg. 1996;61:674-8.

40. Kuecuek O, Mantouvalou L, Klemz R, Kotsch K, Volk HD, Jonas S, et al. Significant reduction of proinflammatory cytokines by treatment of the brain-dead donor. Transplant Proc. 2005;37:3878.

41. Folkesson HG, Norlin A, Wang Y, Abedinpour P, Matthay MA. Dexamethasone and thyroid hormone pre-treatment upregulate alveolar epithelial fluid clearance in adult rats. J Appl Physiol. 2000;88:416-24

42. Noda M, Suzuki S, Tsubochi H, Sugita M, Maeda S, Kobayashi $\mathrm{S}$, et al. Single dexamethasone injection increases alveolar fluid clearance in adult rats. Crit Care Med. 2003;31:1183-9. 